\title{
Effect of ethanol on magnesium excretion
}

\author{
M. DICK, R. A. EVANS, AND LYAL WATSON \\ From the Medical Unit, University College Hospital, London
}

SYNOPSIS The effect of ethanol on magnesium excretion was studied in three normal subjects. It $\overrightarrow{\vec{\omega}}$ was found that the ingestion of $2 \mathrm{ml}$ ethanol $/ \mathrm{kg}$ body weight produced a marked immediate increase in urinary magnesium excretion, but there was no significant effect on overall magnesium balance? when this amount was taken daily for eight days.

It has been shown that giving ethanol may increase urinary magnesium excretion (Kalbfleisch, Lindeman, Ginn, and Smith, 1963) and patients suffering from chronic alcoholism frequently have low plasma magnesium levels (Fankushen, Raskin, Dimich, and Wallach, 1964); in delirium tremens this reduction is still more marked, and the clinical manifestations bear some resemblance to those of severe magnesium depletion. The question arises as to whether alcohol ingestion produces magnesium deficiency and if so by what mechanism. Dunn and Walser (1966) have shown that subjects already depleted of magnesium do not increase magnesium excretion when given 10 ounces of ninety-proof whisky daily. This paper describes the short-term effect of pure ethanol on magnesium balance in three normal subjects.

\section{MATERIAL AND METHODS}

The subjects were healthy male volunteers aged 38,30 , and 37 years and weighing 73,78 , and $75 \mathrm{~kg}$ respectively. The diet was constant during the four four-day periods, but during the third and fourth periods pure ethanol, $2 \mathrm{ml}$ per kg body weight, was ingested each evening well diluted in water. The balances were preceded by a fourday period of stabilization on the constant diet which matched the ordinary intake of each subject. The periods were separated by carmine red markers, and in addition the faecal results were corrected according to the recovery of a known weight of ingested barium sulphate (either 8.0 or $12.0 \mathrm{~g}$ per four days) used as a continuous internal marker as described by Dick (1967).

The immediate effect of ethanol on urinary magnesium excretion was determined by collecting urine every two hours from 8 am to $6 \mathrm{pm}$, then overnight till $8 \mathrm{am}$ the following day. On the day of this test the dilute ethanol was taken in about one hour between 10 and 11 am instead of during the evening. Magnesium estimations were by atomic absorption spectroscopy, as described by MacDonald and Watson (1966).

Received for publication 30 May 1968.

\section{RESULTS}

The magnesium balances are charted in Figure 1. Each subject was in approximate balance throughout the study and no significant change occurred during the ingestion of ethanol. Figure 2 shows the rapid increase in urinary magnesium excretion following ingestion of dilute ethanol. There was no significant increase in magnesium excretion on a control day when a litre of water was taken in about one hour between 10 and $11 \mathrm{am}$.

\section{DISCUSSION}

Hypomagnesaemia has been reported in many disease states (MacIntyre, 1963); it is commonly interpreted as a sign of magnesium deficiency though this interpretation has been proven by balance studies in only a few cases. Magnesium deficiency may occur in chronic alcoholism but the evidence is not really conclusive and its clinical significance has been questioned (Fankushen et al, 1964).

In all three subjects there was a marked immediate increase in urinary magnesium excretion following $\frac{D}{0}$ ethanol consumption (see Fig. 2). However, this subsided after about six hours and was followed by of a decreased excretion, so that the total during the $N$ 24 hours was similar to that during all the other 0 stages of the study. Full metabolic balances (Fig. 1) showed no significant change during the eight days when ethanol was taken.

The intake of ethanol (140 to $160 \mathrm{ml} /$ day) was $\stackrel{\mathbb{D}}{\mathscr{C}}$ considerable and was as much as the volunteers ? could tolerate. However, it was almost certainly a good deal less than that of the alcoholic presenting $\overrightarrow{\mathbb{D}}$ with delirium tremens and profound hypomag- $\frac{\rho}{1}$ nesaemia. Furthermore the dietary intake is much 2 more likely to be inadequate in such chronic alcoholics and it still remains to be seen whether 8 

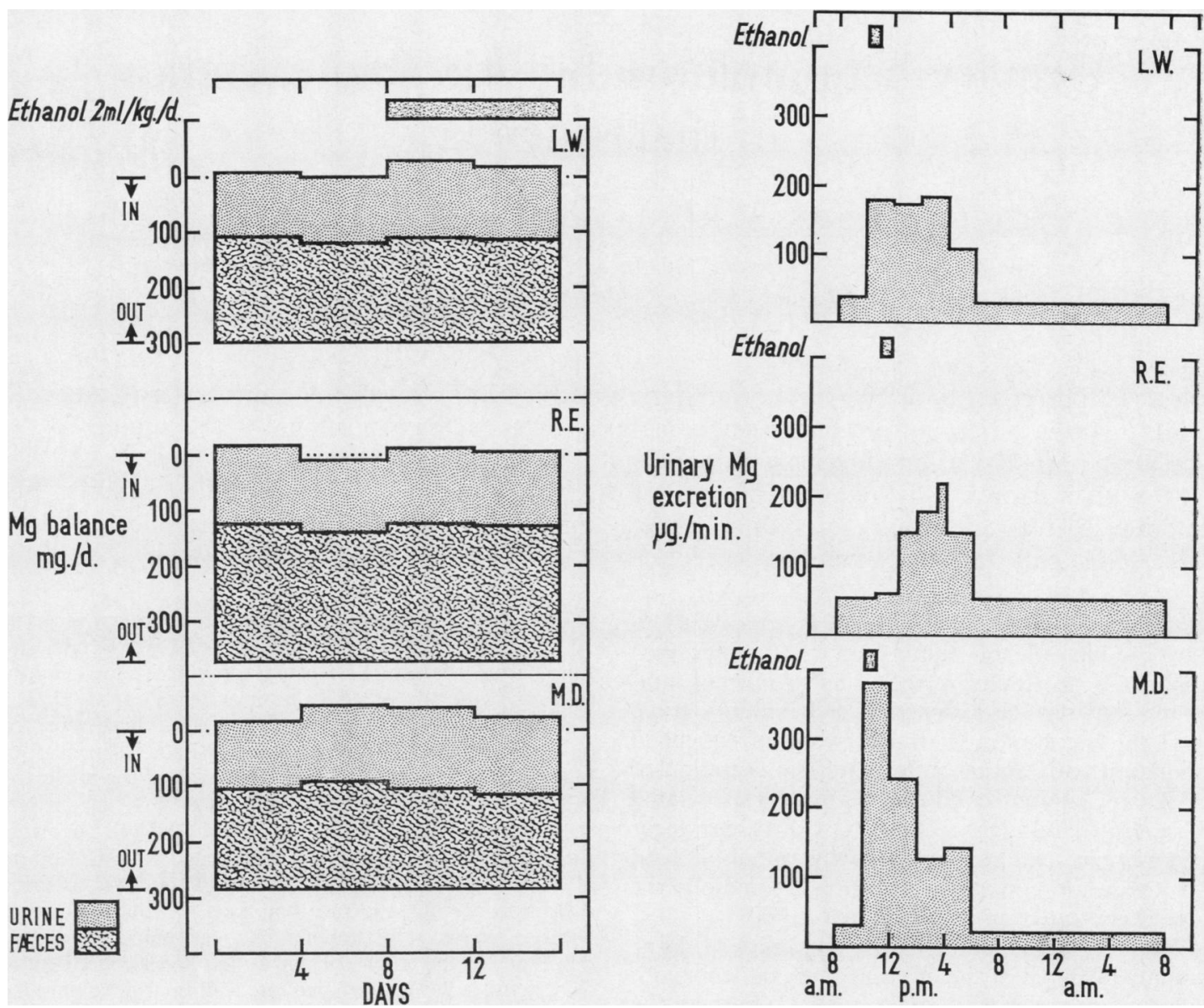

FIG. 1 .

FIG. 2.

FIG. 1. Magnesium balances in three normal volunteers before and during the ingestion of ethanol.

FIG. 2. Urinary magnesium excretion in the three subjects on one day when the ethanol was taken between 10 and $11 \mathrm{am}$.

very large doses taken over most of the waking hours rather than in one short period could produce a sufficiently prolonged stimulus for magnesium excretion to cause depletion.

We are grateful to the dietitians and nurses of the metabolic ward for their assistance and to Professor C. E. Dent for advice in preparing this paper.

\section{REFERENCES}

Dick, M. (1967). J. clin. Path., 20, 216.

Dunn, M. J., and Walser, M. (1966). Metabolism, 15, 884.

Fankushen, D., Raskin, D., Dimich, A., and Wallach, S. (1964). Amer. J. Med., 37, 802.

Kalbfleisch, J. M., Lindeman, R. D., Ginn, H. E., and Smith, W. 0. (1963). J. clin. Invest., 42, 1471.

MacDonald, M. A., and Watson, L. (1966). Clin. chim. Acta, 14, 233. MacIntyre, I. (1963). J. chron. Dis., 16, 201. 